\title{
Prophylactical Low Dose Whole-Liver Irradiation Inhibited Colorectal Liver Metastasis by Regulating Hepatic Niche in Mice
}

This article was published in the following Dove Press journal: OncoTargets and Therapy

\author{
Lu Wang' \\ Yinan Sun ${ }^{2}$ \\ Xiaoxiao Luo' \\ Hu Han' \\ Han Yin' \\ Ben Zhao' \\ Xinyi Chen' \\ Qianqian $\mathrm{Yu}^{\prime}$ \\ Hong Qiu' \\ Xianglin Yuan \\ 'Department of Oncology, Tongji \\ Hospital, Tongji Medical College, \\ Huazhong University of Science and \\ Technology, Wuhan, Hubei, People's \\ Republic of China; ${ }^{2}$ Department of \\ Cardiology, Tongji Hospital, Tongji \\ Medical College, Huazhong University of \\ Science and Technology, Wuhan, Hubei, \\ People's Republic of China
}

Background: The liver is the most common target for metastatic colorectal cancer. Changes of the local hepatic niche due to hepatic diseases such as cirrhosis decrease the incidence of colorectal cancer liver metastasis. Hepatic niche heterogeneity could influence the risk of hepatic metastasis.

Materials and Methods: We simulated changes of the hepatic niche via prophylactical liver irradiation with a safe dose of 6 Gy. GEO dataset and GO analysis revealed a difference in the expression of matrix metalloproteinase 1 (MMP1) in primary colorectal cancer versus liver metastasis, as well as synchronous versus metachronous liver metastasis. Western blotting, Immunofluorescence and qRT-PCR were conducted to measure protein expressions, location and RNA expressions. Colony formation, wound-healing, transwell assays experiments were performed to determine the malignant biological properties of colorectal cancer cells. shRNA transfection was used to conduct stable transfected cell lines.

Results: Tissue inhibitor of metalloproteinases 1 (TIMP1) expression was significantly higher in metastases lesions than primary tumors. In vivo, TIMP1 expression in the hepatic niche increased after a safe dose of 6 Gy irradiation, along with MMP1 decreased, leading to collagen fiber deposition and impairment of hepatic microcirculation. In vitro, irradiated hepatic stellate cells-conditioned media reduced the migration and clone formation ability of colon cancer cells SW480 and HCT116. Low TIMP1 expression in hepatic stellate cells reduced tumor cell invasion and migration.

Conclusion: Prophylactical 6 Gy whole-liver irradiation could inhibit colorectal cancer liver metastasis by regulating TIMP1/MMP1 balance in the hepatic niche before liver metastatic lesion formed.

Keywords: prophylactical liver irradiation, colorectal liver metastasis, hepatic niche, metallopeptidase inhibitor-1

\section{Background}

The primary cause of disease progression and death for patients with colorectal cancer is colorectal cancer liver metastasis (CRLM). CRLM occurs in more than $25 \%$ of patients diagnosed with primary colorectal cancer, ${ }^{1,2}$ and half of the patients would be affected by CRLM over the full course of colorectal cancer. ${ }^{1,3,4}$ Moreover, liver metastasis may occur in more than $80 \%$ of CRLM patients while the primary lesion is still clinically undetectable $\left(0.0001-1 \mathrm{~cm}^{3}\right) .^{5}$ Thus, prophylactical intervention as early as possible after primary diagnosis is the most promising method to reduce the incidence of CRLM and mortality of colorectal cancer patients with a high risk of CRLM.
Correspondence: Xianglin Yuan; Hong Qiu

Department of Oncology, Tongji Hospital Tongji Medical College, Huazhong University of Science and Technology, Jie Fang Road 1095, Wuhan, Hubei Province, People's Republic of China Email yuanxianglin@hust.edu.cn; tjqiuhong@163.com 
The heterogeneity of the hepatic niche is a critical factor for the occurrence of CRLM. A large number of clinical studies ${ }^{6-10}$ have shown that previous liver diseases such as hepatitis, liver fibrosis, and cirrhosis can change both the hepatic niche and local immunity to reduce the incidence of CRLM. Hepatic stellate cells (HSCs) in the liver microenvironment were shown to be involved in chronic liver diseases and the progression of liver damage to eventual cirrhosis. ${ }^{11,12}$ Moreover, matrix metalloproteinases (MMPs) and tissue inhibitors of MMPs (TIMPs) secreted by HSCs are the most important factors involved in extracellular matrix (ECM) remodeling. Imbalance of the TIMP1/MMP1 ratio can accelerate liver fibrosis, and studies have shown that high TIMP1 and low MMP1 expression in liver fibrosis leads to ECM accumulation and restoration of the hepatic lobule structure. ${ }^{13,14}$ Furthermore, MMP levels indicated the strength of correlation between cancer cells and the liver microenvironment, whereas differences in expression of specific MMPs and TIMPs were associated with susceptibility to chemotherapy in CRLM. ${ }^{15}$ Therefore, local interventions that adjust the expression of MMPs/TIMPs in the hepatic niche before CRLM might be a novel method to prevent its development.

Local intervention by liver irradiation is widely used for the treatment of primary and secondary liver cancer. It works both through directly killing tumor cells and affecting HSC status and ECM distribution in the hepatic niche, ${ }^{16}$ which suggests that it can prevent CRLM by modifying colorectal cancer cell anchorage to the liver. As such, whole-liver low dose irradiation may be a novel treatment option to prevent CRLM by regulating the balance of TIMP1/MMP1 in the liver microenvironment. The aim of our study was to provide evidence to uncover the mechanism by which it may decrease the incidence of CRLM. To our knowledge, this is the first report that prophylactical whole-liver irradiation at a safe dose can reduce the incidence of CRLM.

\section{Materials and Methods}

\section{Patients}

Basic clinical information was collected for patients who underwent primary colorectal cancer radical resection in Tongji hospital from January 2015 to January 2017, including gender, age, clinical adjuvant therapy, initial tumor site, disease stage, degree of differentiation, and past history of liver disease. According to the literature, approximately $40 \%$ of patients exhibit recurrence and metastasis after radical resection, with the majority occurring within two years and accounting for $80 \%$ of all diagnosed patients. ${ }^{17-19}$ Therefore, in this study, we assessed the occurrence of liver metastasis within two years after radical resection. The patients that did not develop liver metastasis after two years were defined as non-metastasis patients. This study was approved by the ethics committee of Tongji Hospital and was conducted according to the Declaration of Helsinki and the Good Clinical Practice Guidelines of the International Conference on Harmonization, all patients provided informed consent.

\section{Animal Experiments}

Six-week-old C57/BL6J male mice (Huafukang Bioscience Co., Beijing) were selected and raised in a specific pathogen-free (SPF) animal laboratory. Mice were separated into control and experimental groups. The experimental group was immobilized on a small animal irradiator board, following abdominal anesthesia with sodium pentobarbital $(1 \%, 50 \mathrm{mg} / \mathrm{kg})$. The upper costal and sub-umbilical areas were covered with a lead plate, which exposed only the whole-liver area to irradiation of 6 Gy from a small animal irradiating apparatus (Rad Source, RS2000). The control group was anesthetized using the same method, without irradiation, then fed in the same SPF animal laboratory. At 8 weeks following irradiation, on an aseptic operating table, the CRLM model was initiated by injecting MC38 cells transfected with luciferase (Cell Bank of the Chinese Academy of Sciences, Shanghai, China) into the spleen of both groups. After 4 weeks, hepatic metastatic lesions were detected via in vivo imaging (Spectral Instrument Imaging, Lago X). The small animal B ultrasonic (Vevo1100, Fujifilm Visualsonics, Canada) device was also used to assess the number and size of hepatic metastases inside the liver. Blood samples from each group were collected via orbital blood collection. The liver function index was determined by transaminase, bilirubin, and albumin concentrations measured by ELISA according to the kits' protocols. Liver and spleen tissues were embedded and analyzed by H\&E staining and Masson's trichrome staining, as well as MMP1 (Abclone \#2,758,853) and TIMP1 immunohistochemical staining (Absin \#131,990). The animal experiments were carried out according to the National Institutes of Health animal use guidelines and approved by Tongji Hospital Institutional Review Board of Experimental Animals. 


\section{GEO Datasets}

We searched the National Center for Biotechnology Information Gene Expression Omnibus (GEO) database and selected gene expression data from the following studies related to CRLM: GSE89393, GSE92914, GDS4396, and GDS3501. The data were analyzed and genes that were found to have statistically significant differences in expression in all datasets were identified. Moreover, Gene Ontology (GO) analysis was performed to identify the most enriched signaling pathways in CRLM

\section{Cell Lines}

Two types of human colon cancer cells and hepatic stellate cells (Cell Bank of the Chinese Academy of Sciences, Shanghai, China) were cultured using Dulbecco's modified Eagle's medium (DMEM, HyClone) or Roswell Park Memorial Institute medium (RPMI, HyClone) 1640, respectively. The media were supplemented with $10 \%$ fetal calf serum (Gibco) and 1\% penicillin/streptomycin (Gibco 15,140-122). Cell lines were washed with phosphate buffer saline (PBS) (HyClone), and the medium was replaced with fresh culture medium when the cells were passaged and seeded on plates (BEAVER, Cat. 40,106). Cells were stored at $-80^{\circ} \mathrm{C}$ in DMSO (MP, CAS 67-68-5) and culture medium. A crystal violet staining solution (Solarbio, G1063) was used for cell counting. The small animal irradiating apparatus was used for cell irradiation.

\section{Wound-Healing Assay}

A total of $5 \times 10^{5}$ colorectal cancer cells were seeded on a 6-well plate (BEAVER, Cat. 40,106) and cultured overnight. Subsequently, a vertical scratch was drawn using a pipette tip, and conditioned culture medium was added after washing 3 times with PBS. The dimensions of each scratch were measured at 5 positions at $0 \mathrm{~h}$ and $24 \mathrm{~h}$. The distances (D) were calculated and compared by applying the formula: $\mathrm{D}=\mathrm{D}_{0 \mathrm{~h}}-\mathrm{D}_{24 \mathrm{~h}}$.

\section{Clone Formation Assay}

After inoculating 200 cancer cells in each 6-well plate and leaving them to attach overnight, the medium was replaced with conditioned culture medium. After 2 weeks, when visible clones appeared in the dish, the medium was discarded, and the cells were washed 3 times in PBS. Subsequently, $2 \mathrm{~mL}$ of pure methanol (SCR, CAS 67-561) was added, and the cells were fixed for $15 \mathrm{~min}$. The fixing solution was discarded, and a $0.1 \%$ crystal violet staining solution was added. Cells were stained for $30 \mathrm{~min}$, washed 3 times with PBS and then air-dried.

\section{Transwell Assay}

The surface of the upper chamber of the Transwell disk was coated with a 1:8 dilution of $50 \mathrm{mg} / \mathrm{L}$ Corning Matrigel (BD, \#354,230), and then air-dried at $4{ }^{\circ} \mathrm{C}$. A total of $50 \mu \mathrm{L}$ of serum-free culture medium was added to each well and incubated for $30 \mathrm{~min}$ at $37^{\circ} \mathrm{C}$. The culture medium was discarded by centrifugation after the digestion. The cells were washed 3 times with PBS and suspended again with the conditioned medium. Cell density was adjusted to $5 \times 10^{5}$. A volume of $200 \mu \mathrm{L}$ of cell suspension was added to the upper chamber and $500 \mu \mathrm{L}$ of complete medium was added into the lower chamber. The supernatant was discarded, and the cells were washed with PBS 3 times. Subsequently, the cells were fixed with $2 \mathrm{~mL}$ of pure methanol for $15 \mathrm{~min}$. The fixing solution was discarded, and $0.1 \%$ crystal violet staining solution was added. Cells were stained for 30 min, washed with PBS 3 times, and then air-dried. A Leica DC 300F orthostatic microscope was used to observe and image 5 fields.

\section{Immunofluorescence}

After cancer cells adhered to the culture slides, conditioned medium was added to the culture for $48 \mathrm{~h}$. The medium was then discarded, and the cells were washed with PBS 3 times, each time for $3 \mathrm{~min}$. The slide was fixed with 4\% paraformaldehyde (Yeasen, 36314ES60) for 15 min and then washed with PBS 3 times, each time for 3 min. Cells were permeabilized with $0.5 \%$ Triton X-100 (SIGMA, T8787) diluted in PBS (PBS-T) at room temperature for $20 \mathrm{~min}$ (this step was omitted if the antigen was expressed on the cell membrane). The cells were then washed with PBS 3 times, each time for $3 \mathrm{~min}$, and dried using a blotting paper. Goat serum (Abcam, ab7481) was added to the slides and sealed at room temperature for 30 min. The blocking solution was discarded, and a drop of PBS-T diluted primary antibody (Abcam, \#EP1247Y) was added to each slide and incubated overnight at $4^{\circ} \mathrm{C}$. Slides were then washed with PBS-T 3 times, each time for 3 min. After drying the slides, PBS-T diluted fluorescent secondary antibody (Cell Signaling Technology, \#4414) was added and incubated at room temperature in the dark for $1 \mathrm{~h}$. Slides were subsequently washed with PBS-T 3 times, each time for $3 \mathrm{~min}$ in the dark. DAPI (Wuhan 
promoter Bio-Tech co., China) was added to the slide and incubated in the dark for $5 \mathrm{~min}$, and then washed with PBS-T 3 times, each time for $5 \mathrm{~min}$. Slides were sealed using a sealing liquid containing an anti-fluorescence quenching agent, and then the slides were observed and imaged using a fluorescence microscope (LSM780, Carl Zeiss, Germany).

\section{Western Blotting}

The protein extract was obtained from cells and separated by NuPAGE 10\% Bis-Tris Gel Electrophoresis, and then transferred to polyvinylidene difluoride (PVDF) membranes (Immobilon, Cat IPVH00010). The membrane was incubated in 10\% bovine serum albumin (Solarbio, A8010) for $1 \mathrm{~h}$ in tris-buffered saline with $0.1 \%$ Tween 20 (TBS-T), then incubated with primary antibodies (Cell Signaling Technology, \#8946, 54,376, 68,463) in 10\% nonfat dry milk at $4^{\circ} \mathrm{C}$. After washing with TBS-T 3 times, the membrane was incubated with the second antibody (diluted according to protocol with $10 \%$ nonfat dry milk in TBS-T) for $1 \mathrm{~h}$ at room temperature. Horseradish peroxidase (HRP)-conjugated anti-rabbit IgG (Cell Signaling Technology, \#7074) or HRP-conjugated antimouse IgG (Cell Signaling Technology, \#7076) was used as secondary antibodies. Proteins were visualized using Chemiluminescent Substrate (Thermo SuperSignal West Pico PLUS). All experiments were independently performed 3 times.

\section{shRNA Transfection}

MC38 cells were infected with a lentivirus (Genechem, Shanghai, China) containinga luciferase gene in media with polybrene and cultured in a $37^{\circ} \mathrm{C}$ incubator with $5 \%$ $\mathrm{CO}_{2}$ to stably express the luciferase. Puromycin (Sigma, USA) was used to select infected cells with puromycin resistance. Vectors containing a TIMP1-based short hairpin RNAs (shRNA) sequence (sh-TIMP1) and scrambled shRNA were purchased from Genechem, Shanghai, China.
The scramble shRNA was used as the normal control (shNC). According to the manufacturer's instructions, stable transfected cell lines were selected by puromycin treatment of the culture medium for the indicated time. Transfection efficiency was assessed by Western blotting and quantitative real-time polymerase chain reaction (qRTPCR).

\section{qRT-PCR}

Total RNA was extracted by Trizol (Sigma), and then reverse transcribed into cDNA by PrimeScript ${ }^{\mathrm{TM}}$ RT Master Mix (Takara). qRT-PCR was conducted using SYBR Green qPCR Master Mix (MedChemExpress). Expression levels were normalized with GAPDH and calculated using the $2^{-\Delta \Delta \mathrm{Ct}}$ index. The specific primers used for RT-PCR are shown in Table 1.

\section{Statistical Analysis}

Statistical analysis was performed using the SPSS software (version 16.0, SPSS Inc, Chicago, IL) and GraphPad Prism 5.0 (GraphPad, San Diego, CA, USA). Descriptive statistics were used for basic characteristics of the patients. Multiple regression analysis was used to find the clinical factors related to CRLM. Student's $t$-test was performed to calculate and analyze the number of hepatic metastases, liver function index, $\mathrm{H}$ score of IHC, transwell cells, and clone formation. Gray analysis, quantitative fluorescence analysis were performed, and migration distance was measured using the Image $\mathrm{J}$ software (National Institutes of Health, USA), $* P<0.05, * * P<0.01, * * * P<0.001, * * * * P<0.0001$.

\section{Results}

\section{Hepatic Disease and CRLM}

A total of 246 colorectal cancer patients who had undergone radical surgery from January 2015 to January 2017 were included in this study, including 165 patients without liver metastasis and 81 patients with liver metastases at two years post-resection. Tumor stage, degree of

Table I Primers Used for qRT-PCR

\begin{tabular}{|l|l|l|}
\hline Target & Forward Primer & Reverse Primer \\
\hline MMPI & CAGATGCTGAAACCCTGAA & CAGATGTGTTTGCTCCCA \\
TIMPI & TTCCAGTCCCGTCACCTT & CAGGCTTCAGCTTCCACTC \\
TIMP2 & TTGCAGGAGGAATCGGT & ACAGGCAAGAAGCAATGG \\
TIMP3 & GGCACCTGAAGTTTCCCT & GCCCTCTGACATCGCTT \\
TIMP4 & TGAGTATGCAGGTCGGAAG & CCCCAGGCTTTATGAGGT \\
GAPDH & ATCATCAGCAATGCCTCCT & CATCACGCCACAGTTTCC \\
\hline
\end{tabular}


differentiation, adjuvant therapy, and previous liver disease were shown to be independent risk factors for CRLM in Table 2. A total of 23 patients had a previous liver disease, 18 patients infected hepatitis $\mathrm{B}$, three patients had schistosomiasis and two with unknown causes. Among patients with hepatitis B, seven patients had cirrhosis. Patients with previous liver disease were shown to have a lower incidence of CRLM, suggesting that passive changes of the liver microenvironment due to hepatic diseases could reduce the occurrence of CRLM.

\section{GEO Datasets Related to CRLM}

We searched for CRLM-related datasets in the GEO database. GSE89393, GSE92914, GDS4396, and GDS3501 were chosen for GO analysis. Three genes, $M M P 1, A N C A$, and NEGR1, had statistically significant differential expression in all GEO datasets, and were selected for further study. GO analysis of GSE89393 and GSE92914 suggested that genes related to ECM structure constituents and metallopeptidase activity

Table 2 Clinical Data of Patients with Colorectal Cancer After R0 Resection

\begin{tabular}{|c|c|c|c|c|c|}
\hline \multirow{3}{*}{$\begin{array}{l}\text { Age } \\
\qquad \begin{array}{l}\geq 60 \\
<60\end{array}\end{array}$} & \multicolumn{2}{|c|}{$\begin{array}{l}\text { Non-Metastasis } \\
(\mathrm{N}=165)\end{array}$} & \multicolumn{2}{|c|}{$\begin{array}{l}\text { Metastasis } \\
(\mathrm{N}=8 \mathrm{I})\end{array}$} & $\begin{array}{l}\boldsymbol{P} \text {-value } \\
0.36\end{array}$ \\
\hline & 64 & $70 \%$ & 27 & $30 \%$ & \\
\hline & 101 & $65 \%$ & 54 & $35 \%$ & \\
\hline Gender & & & & & 0.72 \\
\hline Male & 104 & $67 \%$ & 52 & $33 \%$ & \\
\hline Female & 61 & $68 \%$ & 29 & $32 \%$ & \\
\hline Primary location & & & & & 0.68 \\
\hline Left colon & 142 & $67 \%$ & 71 & $33 \%$ & \\
\hline Right colon & 23 & $70 \%$ & 10 & $30 \%$ & \\
\hline Stage & & & & & 0.029 \\
\hline II & 80 & $75 \%$ & 26 & $25 \%$ & \\
\hline III & 85 & $61 \%$ & 55 & $39 \%$ & \\
\hline Differentiation & & & & & 0.037 \\
\hline Poor & 26 & $58 \%$ & 19 & $42 \%$ & \\
\hline Moderate & 90 & $65 \%$ & 48 & $35 \%$ & \\
\hline High & 49 & $78 \%$ & 14 & $22 \%$ & \\
\hline Adjuvant therapy & 128 & $75 \%$ & 42 & $25 \%$ & 0.001 \\
\hline RAS state & & & & & \\
\hline Wild & 102 & $65 \%$ & 55 & $35 \%$ & 0.54 \\
\hline Mutation & 63 & $71 \%$ & 26 & $29 \%$ & \\
\hline Hepatic disease & 20 & $87 \%$ & 3 & $13 \%$ & 0.025 \\
\hline
\end{tabular}

were the most enriched in CRLM, except for receptor activity. Compared to the primary colon lesions, there was a statistically significant difference in the expression of TIMP1 in liver metastases. Therefore, MMP1 and TIMP1 were selected for subsequent in vitro analyses (Figure 1).

\section{Prophylactical Liver Irradiation Inhibited CRLM in vivo}

Schematic diagrams of the in vivo study are shown in Figure 2A. The number of hepatic metastasis detected both by small animal B ultrasound and visual inspection was lower in the experimental group (Figure $2 \mathrm{~B}$ and $\mathrm{C}$ ). As well, by in vivo fluorescent imaging of labeled cancer cells, the fold change in fluorescence in the experimental group was lower than in the control group (Figure 2D). After lowdose irradiation of $6 \mathrm{~Gy}$, liver function indicators, such as total and direct bilirubin and AST, of C57/BL6 mice were shown to be slightly, but not significantly, increased (Figure 3A and B). However, ALT was shown to be significantly increased, and mouse weight and albumin levels were found to be significantly decreased (Figure 3A-D). The survival rate of mice in the CRLM model was $100 \%$ at one week after 6 Gy low-dose irradiation, and the median overall survival in the experimental group was higher than that of the control group (Figure 3E). Hematoxylin and eosin (H\&E) staining showed a decrease in the diameter of the hepatic small central veins (Figure 4A) and Masson's trichrome staining showed an increase in the relative collagen area (Figure 4B) in the experimental group. MMP1 expression was significantly decreased and TIMP1 expression was significantly increased in the experimental group (Figure 4C).

\section{Status Changes of HSCs Affected CRLM-Related Phenotypes in vitro}

Schematics of in vitro experiments are shown in Figure 5A. SW480 or HCT116 cells were co-cultured with conditioned medium of LX-2 that had been irradiated at $6 \mathrm{~Gy}$. SW480 cultured in the supernatant of HSCs after 6 Gy irradiation formed a smaller number of clones by clone formation assay, and had impaired mobility in the wound-healing assay. Moreover, in the transwell assay, this experimental group showed fewer migrating cells compared with the control group, which was cultured with non-irradiated HSC supernatant. There were no statistically significant differences in these assays for cancer cells cultured in media from 


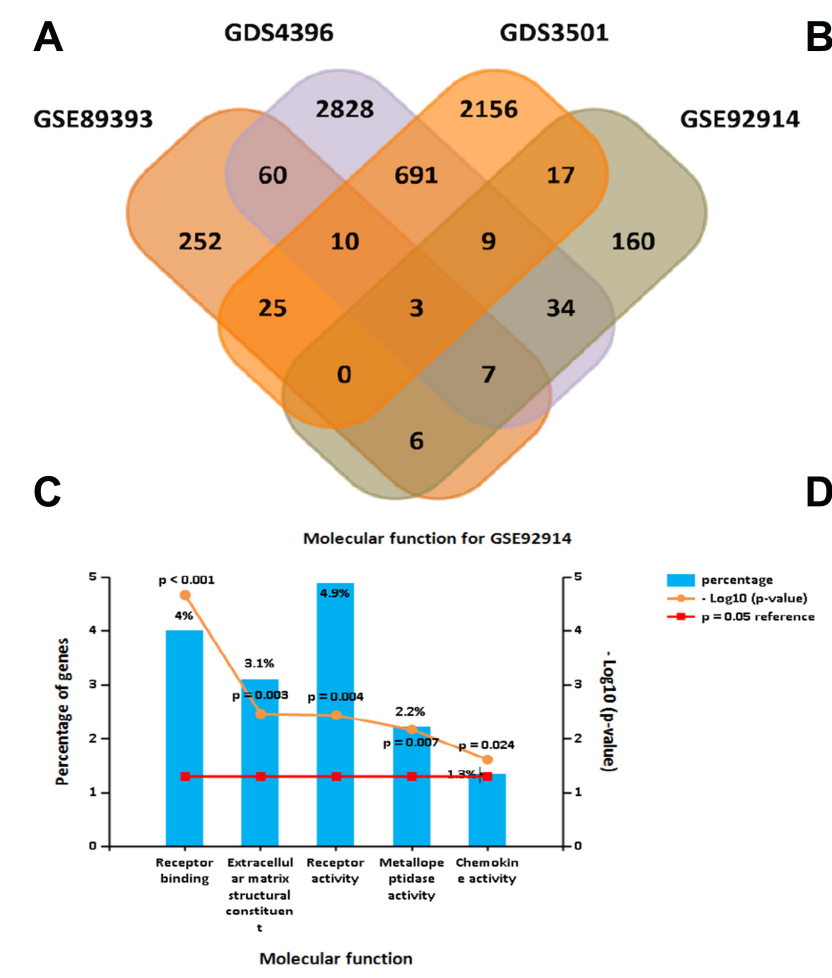

B
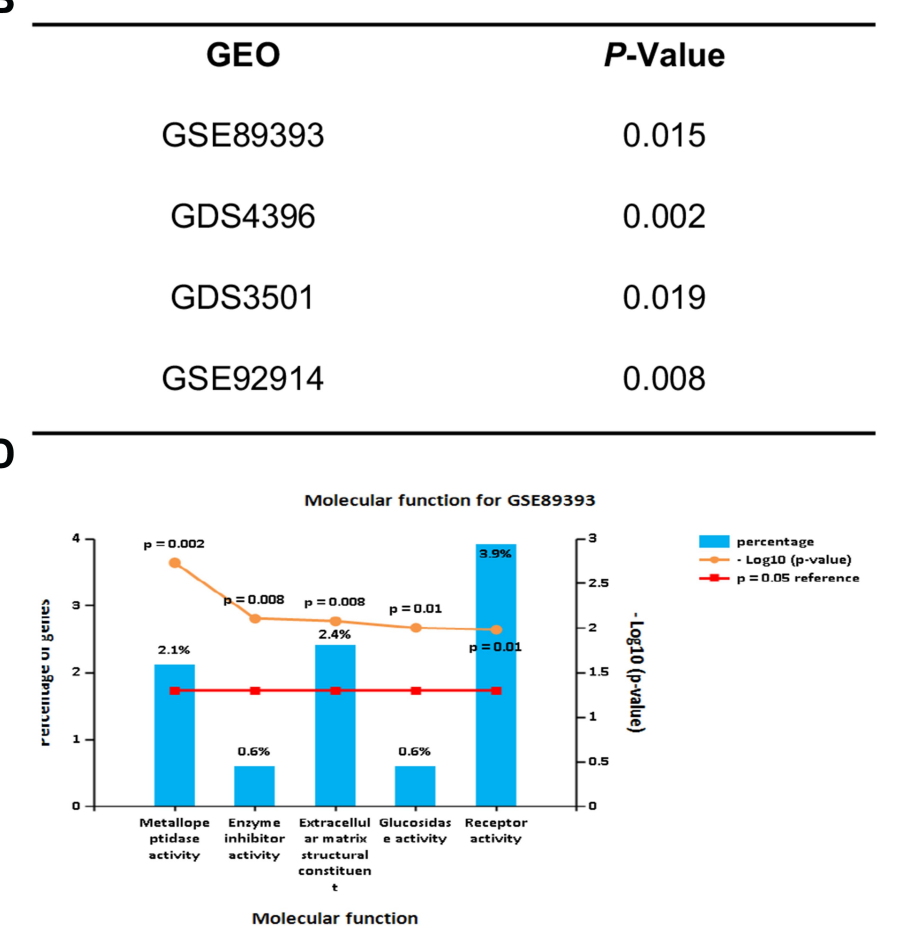

Figure I Colorectal liver metastasis related GEO databases. GSE89393, GSE92914, GDS4396, GDS350I were chosen to analyze the differential expression of proteins between primary colorectal tumors and hepatic metastasis lesions. (A) MMPI, NEGRI, ACAN were identified from the intersection set. (B) P-value of MMPI differential expression. (C) GO analysis of molecular function $(P<0.05)$. Extracellular matrix structural constituent and metallopeptidase activity are the most significantly enriched, except for receptor activity in GSE92914 and (D) GSE89393.

irradiated TIMP1-shRNA HSCs (Figure 5). HSC supernatant after 6 Gy irradiation applied to colorectal cancer cells reduced MMP1 expression and increased TIMP1 expression. There were no significant changes observed when TIMP1-shRNA HSCs were used (Figure 6A). Western blotting indicated that the expression of $\alpha$ SMA and TIMP1 increased, and that of MMP1 decreased, in HSCs after irradiation (Figure 6B). By qRT-PCR, cells cultured in supernatant from 6 Gyirradiated HSCs had lower $M M P 1$ expression and higher TIMP1 expression, but no change in TIMP2-4 expression (Figure 6C). Laser confocal immunofluorescence showed that MMP1 expression in the cytoplasm of colorectal cancer cells was lower when they were treated with supernatant from 6 Gy-irradiated HSCs. There was no significant difference in the expression of MMP1 in cells cultured in the supernatant of 6 Gy-irradiated TIMP1-shRNA HSCs (Figure 6D). The mechanism that whole-liver 6 Gy irradiation might prevent CRLM is shown in Figure 7.

\section{Discussion}

This study found that remodeling of the hepatic niche due to previous liver diseases, such as cirrhosis, hepatitis $\mathrm{B}$, and schistosomiasis reduced the incidence of CRLM in colorectal cancer patients. Therefore, we suggest the adoption of a prophylactical novel local intervention, whole-liver irradiation, to change the hepatic niche and reduce CRLM without disturbing normal liver function. The results of this study confirmed that $6 \mathrm{~Gy}$ liver irradiation significantly reduced the incidence of CRLM. GO analysis of four clinical datasets associated with CRLM found that, compared to primary colorectal cancer, CRLM gene expression was enriched for ECM structure constituents and metallopeptidase activity MMP1/TIMP1, except for receptor activity. We found that after irradiation, HSC supernatant reduced the invasion and clone formation ability of colon cancer cells through increased TIMP1 and decreased MMP1 expression. This suggests that prophylactical liver irradiation adjusts the balance of MMP1 and TIMP1 
A

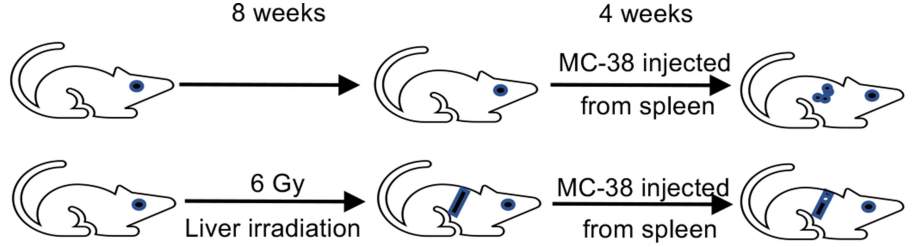

B

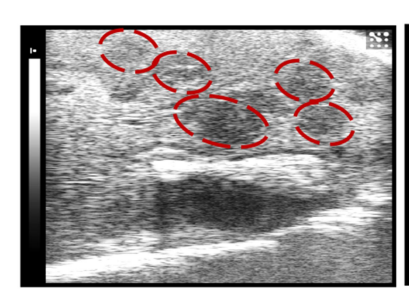

Control

C

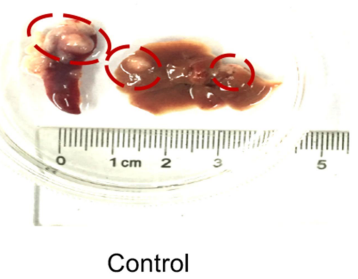

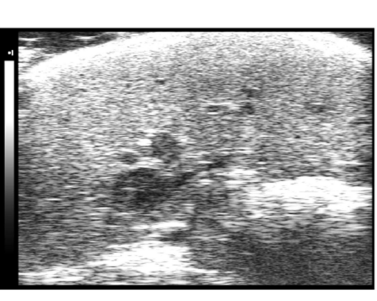

6 Gy

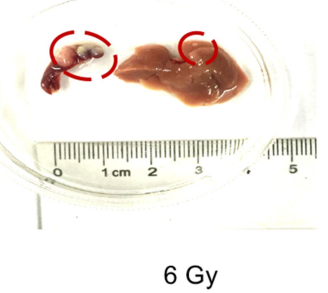

D

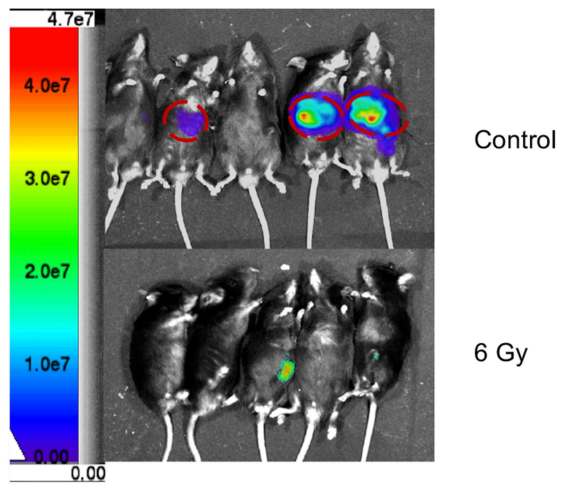

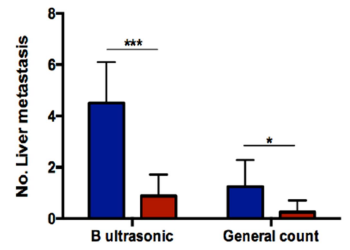

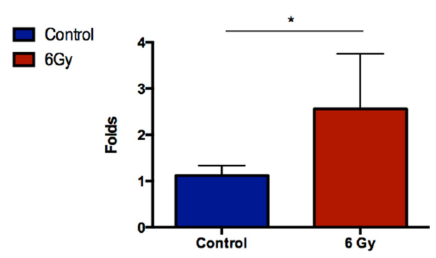

Figure 26 Gy liver irradiation prevented hepatic metastasis in vivo. (A) Schematic diagrams of the in vivo study. The number of hepatic metastatic lesions was detected by animal ultrasonography (B), general view (C), and an imaging system (D). The red circles in panel (B) indicated the liver metastasis lesion in the hypoechoic area and the red circles in panel $(\mathbf{C})$ indicated the colorectal liver metastatic lesions in liver and spleen. Hepatic metastasis lesions in the control group are more in number than those in the 6 Gy group, as detected by ultrasonography. $* P<0.05, * * * P<0.001$.

expression in colorectal cancer cells and reduces the incidence of CRLM.

Regardless of the targeted organ, pre-clinical metastasis is a common phenomenon, which highlights the importance of early prevention in metastasis. The first step of tumor invasion and metastasis is degradation of the ECM and basement membrane barrier. $^{20}$ Moreover, ECM accumulation due to an MMP1/TIMP1 imbalance is central to the process of hepatic niche remodeling during conditions such as cirrhosis and CRLM. $^{21,22}$ Researchers have shown that for patients with liver fibrosis and cirrhosis, abnormal expression of MMPs and TIMPs leads to excessive pathological deposition of ECM. $^{23,24}$ In this study, 8 weeks after whole-liver irradiation of $6 \mathrm{~Gy}$ in mice, the number of central small veins was reduced, decreasing micro-circulation in the sinus. The most primitive cells in the liver involved in hepatic structure remodeling include activated HSCs with a special geographical position in the space of Disse, which not only secrete $\alpha$-SMA, large amounts of ECM, and high TIMP, but also directly secrete cytokines related to ECM remodeling that feedback on HSCs. ${ }^{25-27}$ TIMP1 is secreted from activated HSCs, hepatocytes, and endothelial cells and could inhibit MMPs expression, especially MMP1. It is mainly located in the central vein and the hepatic portal area. ${ }^{28-31}$ Moreover, high expression of
MMP1 was related to the progression and poor prognosis of patients, as well as the invasion and metastatic ability of cancer cells. ${ }^{32}$ In this study, expression of TIMP1 was increased after 6 Gy irradiation of HSCs, which inhibited MMP1. After the supernatant was applied to colorectal cancer cells, the expression of MMP1 in cancer cells decreased, while the expression of TIMP1 increased, thereby inhibiting colorectal cancer invasion and metastasis.

Whole-liver irradiation dose of 6 Gy was shown to be safe, with no observed radiation-induced liver injuries. Moreover, it was capable of remodeling the hepatic niche. Radiation therapy, such as stereotactic body radiation therapy, is an important method in the treatment of primary or metastatic liver cancer. It not only directly destroys tumor cells but also changes the structure of the liver stroma and immune microenvironment to affect the progression of CRLM. Previous studies in mice have reported no obvious radiation-induced liver injury after 5 Gy irradiation, whereas some mice showed unstable liver injury after 10 Gy irradiation. ${ }^{33}$ Therefore, whole-liver irradiation under $10 \mathrm{~Gy}$ is considered safe for mice. In this study, the total and direct bilirubin and transaminase in mice increased at 8 weeks following 6 Gy whole-liver irradiation. However, there was no statistical difference in the survival prognosis, indicating that whole-liver irradiation at $6 \mathrm{~Gy}$ is safe to 
A

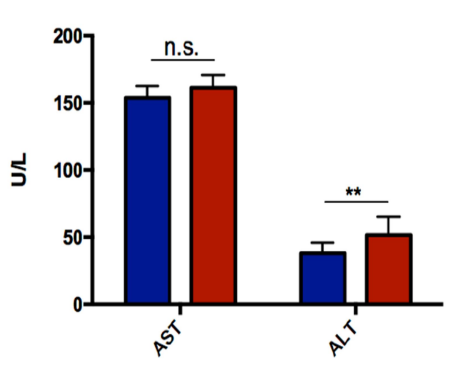

D

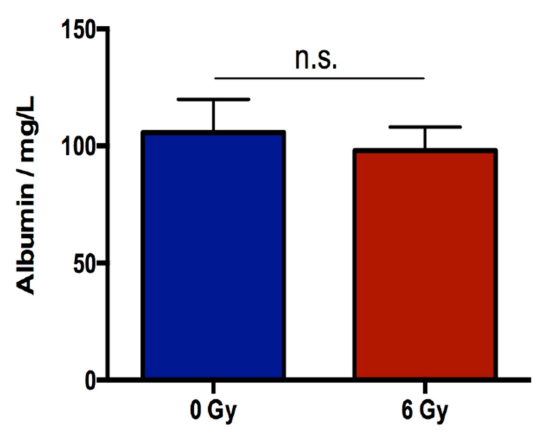

B

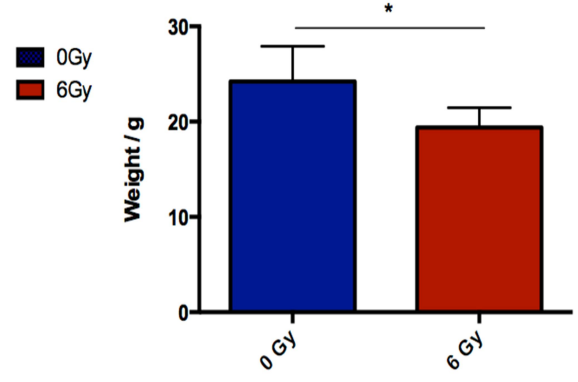

C
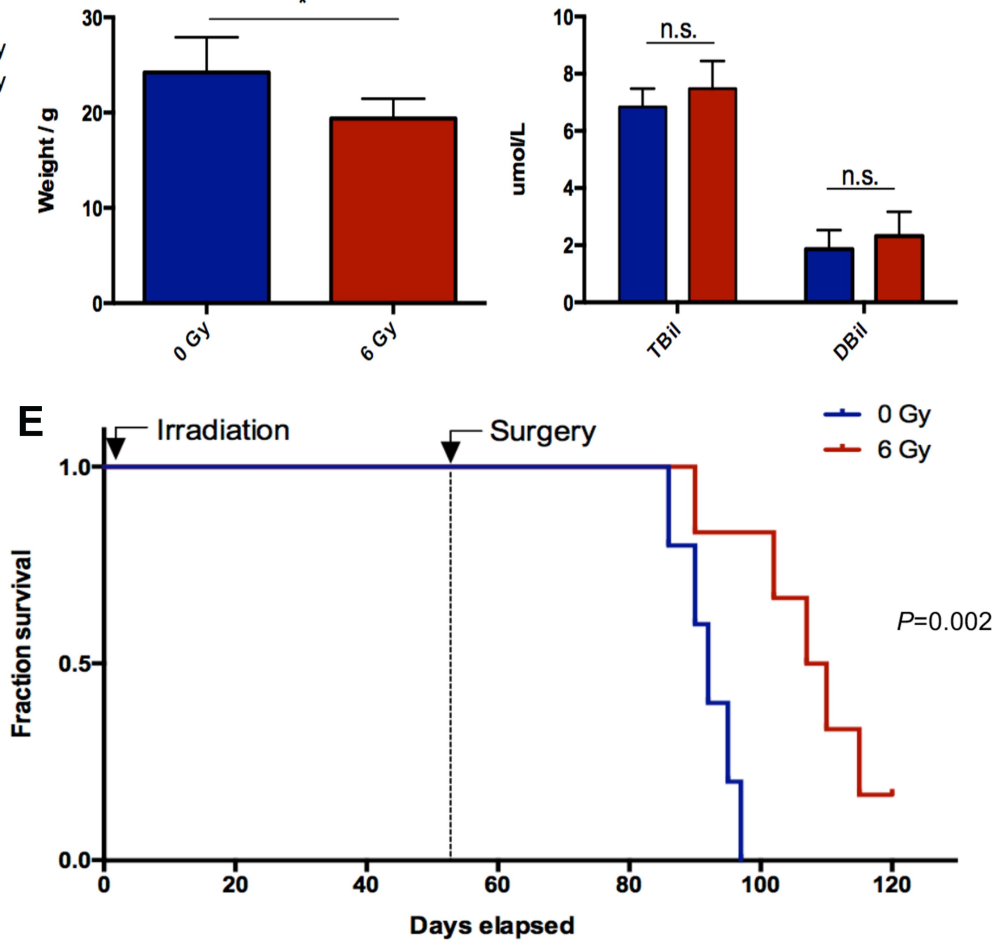

Figure 3 Evaluation of liver function, weight, and overall survival. (A) AST and ALT, (B) weight, (C) direct bilirubin (Dbil) and total bilirubin (Tbil) measurements. (D) Albumin measurements. (E) Overall survival. After 6 Gy low-dose irradiation, liver function indicators of total and direct bilirubin and AST of C57/BL6 mice increased but not significantly. ALT significantly increased whereas the weight of the mice and albumin levels significantly decreased. $* P<0.05, * * P<0.01$.

Abbreviation: n.s., not significant.

A

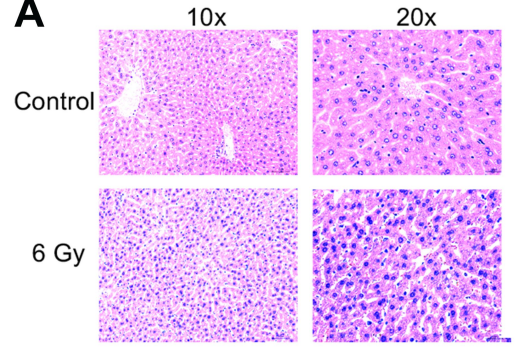

B

$10 x$

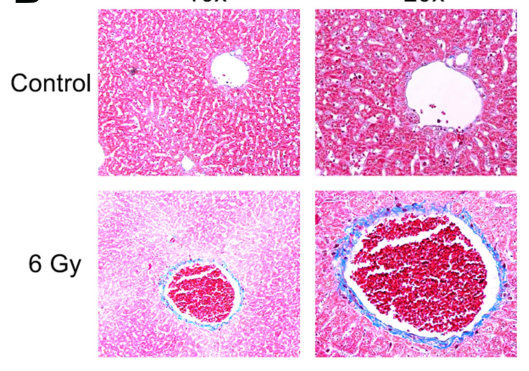

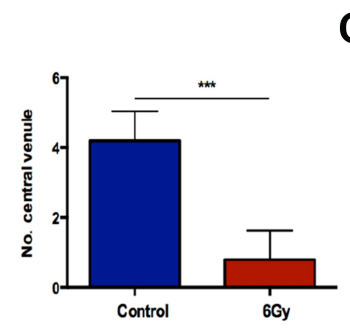

C

$10 x$

\begin{tabular}{ccc}
\multicolumn{2}{c}{ Control } \\
MMP-1 & TIMP-1 & Gy \\
& TIMP-1
\end{tabular}
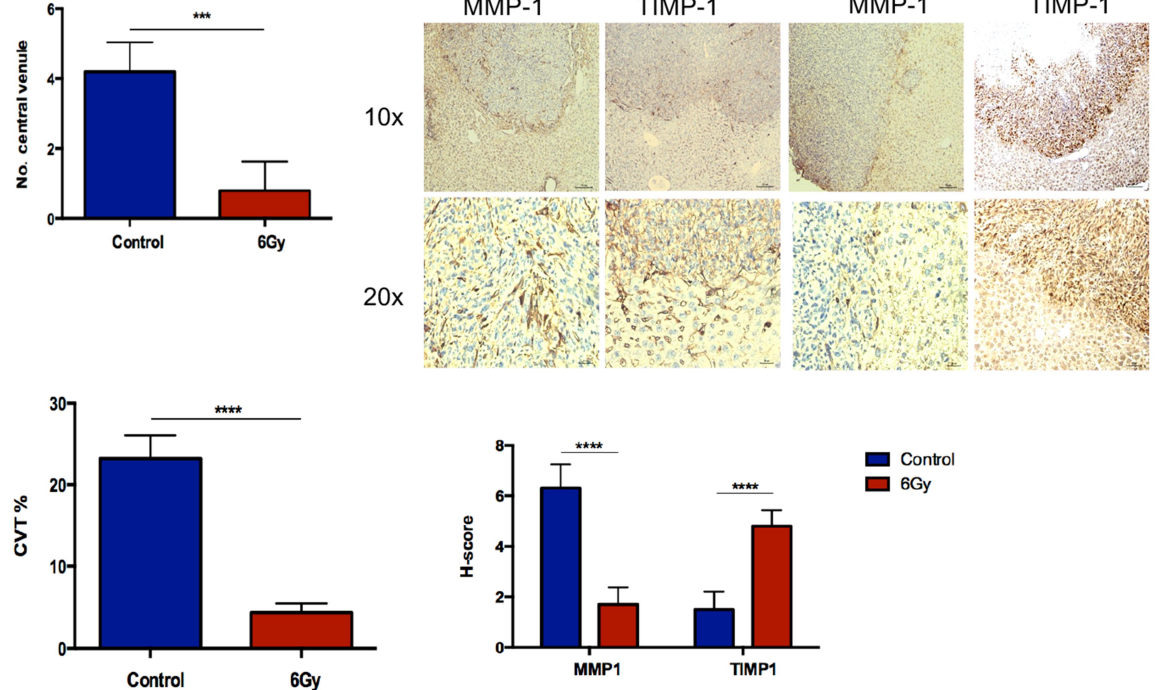

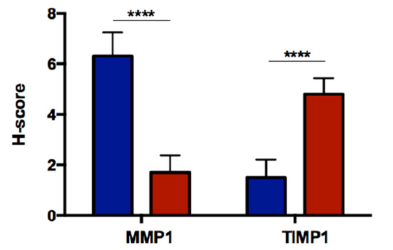

Control
$\square$ 6Gy

Figure 4 HE, Masson's trichrome staining and IHC in liver metastasis. (A) H\&E staining shows decreased diameter of the hepatic small central vein. (B) Masson's trichrome staining shows a relative increase in the collagen area. (C) MMPI expression decreased relative to the control group, and TIMPI expression is shown to be increased. ${ }^{* * *}$ < $<$ 0.001 , $* * * * P<0.0001$. 


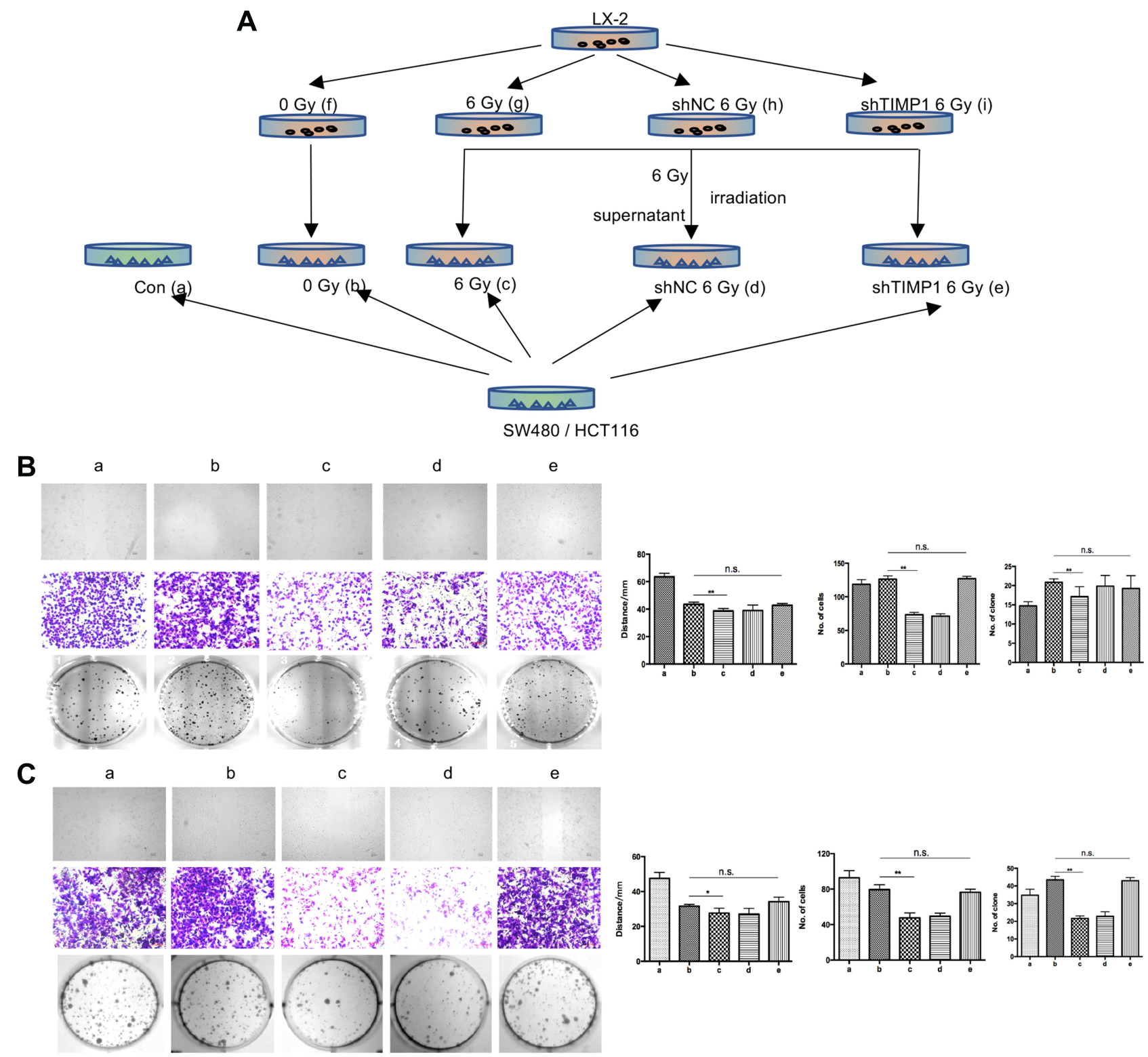

Figure 5 Clone formation assay, wound-healing test, and transwell assay in SW480 and HCTII6. (A) Schematic diagram of in vitro experiments. (B, C) HSC supernatant after 6 Gy irradiation was co-cultured with colon cancer cells. The cells formed a lower number of clones, as observed by the clone formation assay, with decreased mobility as observed in the wound-healing assay. Moreover, the number of migrating cells in the transwell assay was shown to be the lowest. Changes were not significant in cells treated with supernatant from the irradiated TIMPI-shRNA HSCs. $* * P<0.01$.

Abbreviation: n.s., not significant.

preserve normal liver function. Radiation-induced changes in the hepatic sinus include the narrowing of the small central vein and a collagen accumulation gradient, thereby creating a hypoxic niche. ${ }^{34-36}$ In the hepatic sinus near carcinoma, HSCs adjust the balance of TIMP1/MMP1 to form a secondary basement membrane, thereby inhibiting the formation of metastases, which partly explains the lower incidence of CRLM.

\section{Conclusions}

Prophylactical 6 Gy whole-liver irradiation with a safe dose was a novel alternative method to reduce the occurrence of CRLM. Prophylactical 6 Gy whole-liver irradiation disturbed the balance of MMP1/TIMP1 expression in the hepatic niche by changing the status of HSCs, thus leading to resistance to CRLM in mice and inhibit the invasion and metastasis of colorectal cancer cells. 


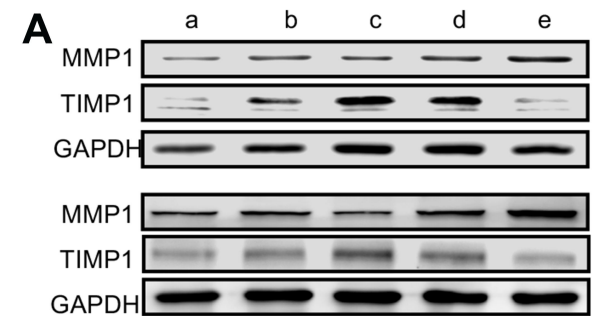

HCT116

GAPDH

SW480

B

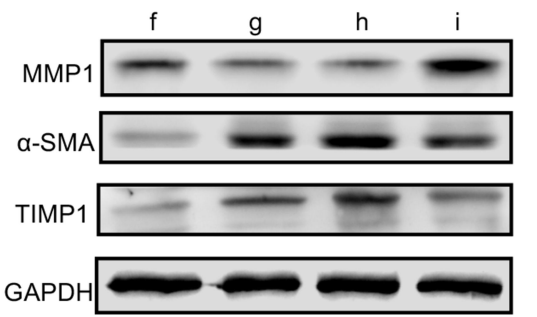

LX-2
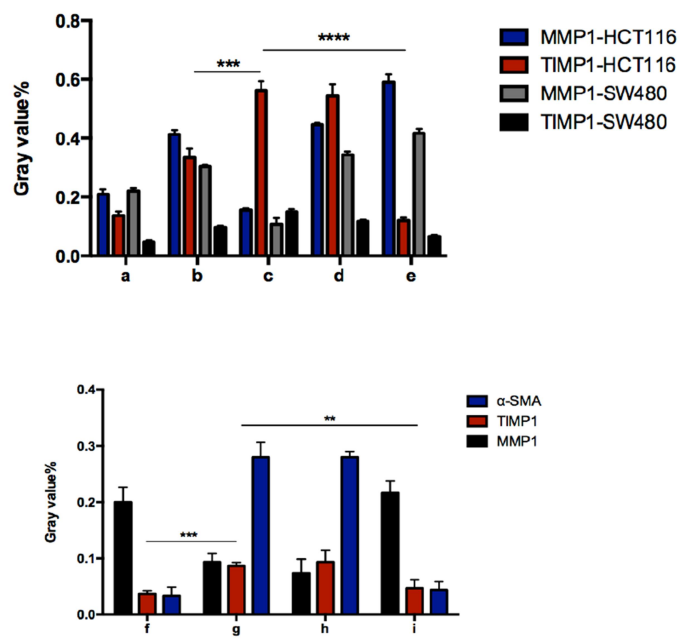

C

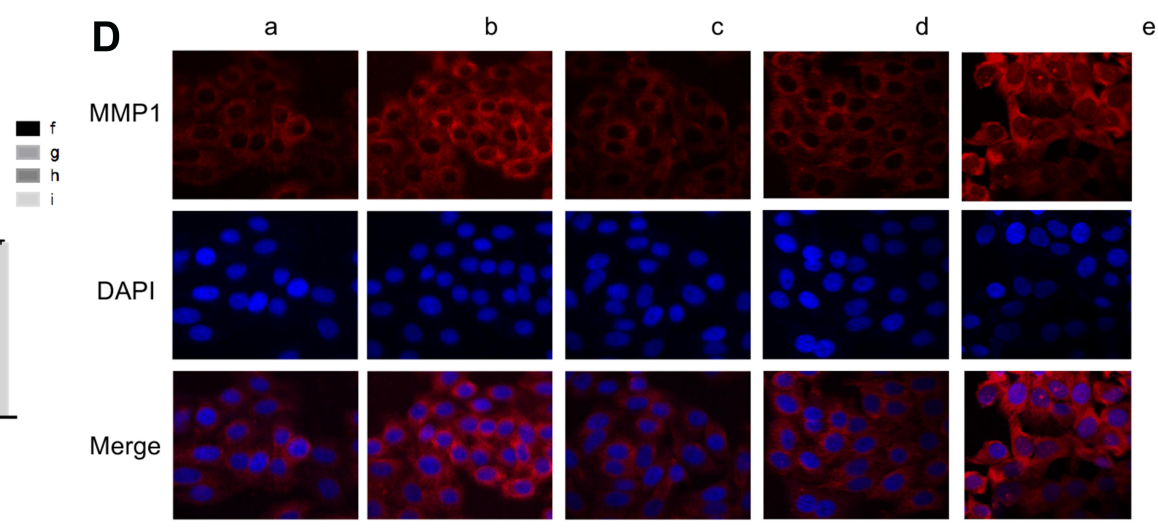

Figure 6 Laser confocal immunofluorescence, PCR and Western blot of TIMPI/MMPI in colon cancer cells. (A) MMPI expression was reduced in the cytoplasm of colon cancer cells cultured in 6 Gy-irradiated HSC supernatant. For the TIMPI-shRNA HSC group, there was no significant difference in the expression of MMPI. (B) $\alpha$-SMA and TIMPI expression level increased and MMPI expression in HSCs decreased after irradiation. (C) qRT-PCR showed that cancer cells grown in supernatant from 6 Gyirradiated HSC had lower MMPI expression and higher TIMPI expression, but no changes were observed in TIMP2-4. (D) HSC supernatant after 6 Gy irradiation cocultured with colon cancer cells was shown to reduce MMPI expression in cancer cells. In the TIMPI-shRNA HSC group, irradiated HSC supernatant did not lead to significant changes. $* * P<0.01$, $* * * P<0.001$, $* * * * P<0.0001$.

Abbreviation: n.s., not significant.

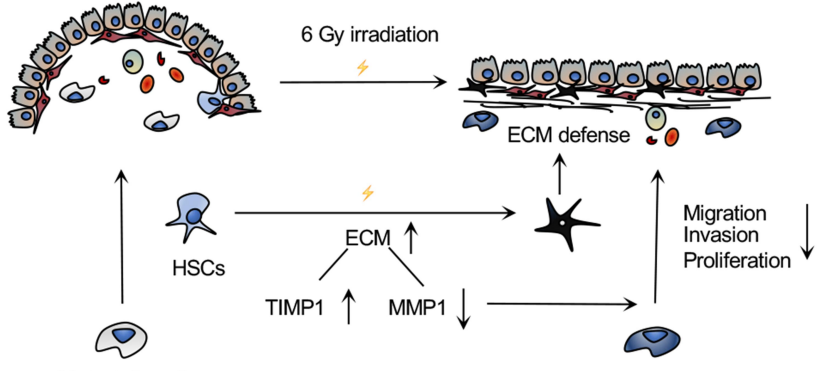

Metastatic cells

Figure 7 Mechanism of action underlying the prevention of CRLM by 6 Gy irradiation of the whole liver. In the hepatic niche, HSCs activated by 6 Gy irradiation secreted more ECM components, which could protect against metastatic cells. Moreover, irradiation induced a TIMPI-high and cirrhosis-like environment, leading to a decrease in MMPI expression and the ability of cancer cells to proliferate and invade, thereby preventing CRLM.

\section{Abbreviations}

CRLM, colorectal cancer liver metastasis; MMP1, matrix metalloproteinase-1; TIMP, tissue inhibitor of MMP; HSCs, hepatic stellate cells; ECM, extracellular matrix; HE, hematoxylin-eosin; DMEM, Dulbecco's modified Eagle medium; RPMI, Roswell Park Memorial Institute medium; PBS, phosphate buffer saline.

\section{Acknowledgments}

We thank the patients and their families in our clinical studies. We also are thankful for the technical support of Weiheng Zhao and Yu Chen. 


\section{Funding}

This study was supported by the "Double First-Class" Construction Project [grant number 540-5001540013] and Tongji hospital Fund [grant number 2019YJJA14].

\section{Disclosure}

The authors declare that they have no competing interests for this work.

\section{References}

1. Hong Y, Rao Y. Current status of nanoscale drug delivery systems for colorectal cancer liver metastasis. Biomed Pharmacother. 2019;114108764. doi:10.1016/j.biopha.2019.108764

2. Gatta G, Capocaccia R, Sant M, et al. Understanding variations in survival for colorectal cancer in Europe: a EUROCARE high resolution study. Gut. 2000;47(4):533-538. doi:10.1136/gut.47.4.533

3. Arnold M, Sierra MS, Laversanne M, Soerjomataram I, Jemal A, Bray F. Global patterns and trends in colorectal cancer incidence and mortality. Gut. 2017;66(4):683-691. doi:10.1136/gutjnl-2015-310912

4. Jones RP, Jackson R, Dunne DF, et al. Systematic review and meta-analysis of follow-up after hepatectomy for colorectal liver metastases. Br J Surg. 2012;99(4):477-486. doi:10.1002/bjs.8667

5. Hu Z, Ding J, Ma Z, et al. Quantitative evidence for early metastatic seeding in colorectal cancer. Nat Genet. 2019;51(7):1113-1122. doi:10.1038/s41588-019-0423-x

6. Cai B, Liao K, Song XQ, Wei WY, Zhuang Y, Zhang S. Patients with chronically diseased livers have lower incidence of colorectal liver metastases: a meta-analysis. PLoS One. 2014;9(9):e108618. doi:10.1371/journal.pone. 0108618

7. Chiou WY, Chang CM, Tseng KC, et al. Effect of liver cirrhosis on metastasis in colorectal cancer patients: a nationwide population-based cohort study. Jpn J Clin Oncol. 2015;45 (2):160-168. doi:10.1093/jjco/hyu178

8. Li Destri G, Castaing M, Ferlito F, Minutolo V, Di Cataldo A, Puleo S. Rare hepatic metastases of colorectal cancer in livers with symptomatic HBV and HCV hepatitis. Ann Ital Chir. 2013;84(3):323-327.

9. Qiu HB, Zhang LY, Zeng ZL, et al. HBV infection decreases risk of liver metastasis in patients with colorectal cancer: a cohort study. World J Gastroenterol. 2011;17(6):804-808. doi:10.3748/wjg.v17.i6.804

10. Seymour K, Charnley RM. Evidence that metastasis is less common in cirrhotic than normal liver: a systematic review of post-mortem case-control studies. Br J Surg. 1999;86(10):1237-1242. doi:10. 1046/j.1365-2168.1999.01228.x

11. de Oliveira da Silva B, Ramos LF, Moraes KCM. Molecular interplays in hepatic stellate cells: apoptosis, senescence, and phenotype reversion as cellular connections that modulate liver fibrosis. Cell Biol Int. 2017;41(9):946-959. doi:10.1002/cbin.10790

12. Higashi T, Friedman SL, Hoshida Y. Hepatic stellate cells as key target in liver fibrosis. Adv Drug Deliv Rev. 2017;12127-12142. doi:10.1016/j.addr.2017.05.007

13. Latronico T, Mascia C, Pati I, et al. Liver fibrosis in HCV monoinfected and HIV/HCV coinfected patients: dysregulation of Matrix Metalloproteinases (MMPs) and their Tissue Inhibitors TIMPs and effect of HCV protease inhibitors. Int J Mol Sci. 2016;17(4):455. doi:10.3390/ijms17040455

14. Magdaleno F, Arriazu E, Ruiz de Galarreta M, et al. Cartilage oligomeric matrix protein participates in the pathogenesis of liver fibrosis. J Hepatol. 2016;65(5):963-971. doi:10.1016/j.jhep.2016.06.003

15. Gentner B, Wein A, Croner RS, et al. Differences in the gene expression profile of matrix metalloproteinases (MMPs) and their inhibitors (TIMPs) in primary colorectal tumors and their synchronous liver metastases. Anticancer Res. 2009;29(1):67-74.
16. Yuan B, Chen Y, Wu Z, et al. Proteomic profiling of human hepatic stellate cell line LX2 responses to irradiation and TGF-beta1. J Proteome Res. 2019;18(1):508-521. doi:10.1021/acs.jproteome.8b00814

17. Alhumaid A, AlYousef Z, Bakhsh HA, AlGhamdi S, Aziz MA. Emerging paradigms in the treatment of liver metastases in colorectal cancer. Crit Rev Oncol Hematol. 2018;13239-13250. doi:10.1016/j. critrevonc.2018.09.011

18. Beretta GD, Milesi L, Pessi MA, Mosconi S, Labianca R. Adjuvant treatment of colorectal cancer. Surg Oncol. 2004;13(2-3):63-73. doi:10.1016/j.suronc.2004.09.008

19. Carlomagno C, De Stefano A, Rosanova M, et al. Multiple treatment lines and prognosis in metastatic colorectal cancer patients. Cancer Metastasis Rev. 2019;38(1-2):307-313. doi:10.1007/s10555-0189748-7

20. Massague J, Obenauf AC. Metastatic colonization by circulating tumour cells. Nature. 2016;529(7586):298-306. doi:10.1038/ nature 17038

21. van Huizen NA, Coebergh van den Braak RRJ, Doukas $M$, Dekker LJM, IJzermans JNM, Luider TM. Up-regulation of collagen proteins in colorectal liver metastasis compared with normal liver tissue. J Biol Chem. 2019;294(1):281-289. doi:10.1074/jbc.RA118. 005087

22. Williamson T, Sultanpuram N, Sendi H. The role of liver microenvironment in hepatic metastasis. Clin Transl Med. 2019;8(1):21. doi:10.1186/s40169-019-0237-6

23. Busk TM, Bendtsen F, Nielsen HJ, Jensen V, Brunner N, Moller S. TIMP-1 in patients with cirrhosis: relation to liver dysfunction, portal hypertension, and hemodynamic changes. Scand $J$ Gastroenterol. 2014;49(9):1103-1110. doi:10.3109/00365521.2014.934910

24. Iredale JP, Pellicoro A, Fallowfield JA. Liver fibrosis: understanding the dynamics of bidirectional wound repair to inform the design of markers and therapies. Dig Dis. 2017;35(4):310-313. doi:10.1159/ 000456581

25. Prestigiacomo V, Weston A, Messner S, Lampart F, Suter-Dick L, Mukhopadhyay P. Pro-fibrotic compounds induce stellate cell activation, ECM-remodelling and Nrf2 activation in a human 3D-multicellular model of liver fibrosis. PLoS One. 2017;12(6): e0179995. doi:10.1371/journal.pone.0179995

26. Kong D, Zhang F, Zhang Z, Lu Y, Zheng S. Clearance of activated stellate cells for hepatic fibrosis regression: molecular basis and translational potential. Biomed Pharmacother. 2013;67(3):246-250. doi:10.1016/j.biopha.2012.10.002

27. Baig MS, Yaqoob U, Cao S, Saqib U, Shah VH. Non-canonical role of matrix metalloprotease (MMP) in activation and migration of hepatic stellate cells (HSCs). Life Sci. 2016;155155-155160. doi:10.1016/j.lfs.2016.04.031

28. Roeb E, Purucker E, Breuer B, et al. TIMP expression in toxic and cholestatic liver injury in rat. J Hepatol. 1997;27(3):535-544. doi:10.1016/S0168-8278(97)80359-5

29. Del Carmen Garcíade León M, Montfort I, Tello ME, et al. Hepatocyte production of modulators of extracellular liver matrix in normal and cirrhotic rat liver. Exp Mol Pathol. 2006;80(1):97-108. doi:10.1016/j.yexmp.2005.03.008

30. Lichtinghagen R, Pietsch D, Bantel H, Manns MP, Brand K, Bahr MJ. The Enhanced Liver Fibrosis (ELF) score: normal values, influence factors and proposed cut-off values. J Hepatol. 2013;59 (2):236-242. doi:10.1016/j.jhep.2013.03.016

31. Robert S, Gicquel T, Victoni T, et al. Involvement of matrix metalloproteinases (MMPs) and inflammasome pathway in molecular mechanisms of fibrosis. Biosci Rep. 2016;36(4):4. doi:10.1042/ BSR20160107

32. Liu X, Zhou ZH, Li W, et al. Heparanase promotes tumor growth and liver metastasis of colorectal cancer cells by activating the p38/MMP1 axis. Front Oncol. 2019;9216. doi:10.3389/ fonc. 2019.00216 
33. Cheng W, Xiao L, Ainiwaer A, et al. Molecular responses of radiation-induced liver damage in rats. Mol Med Rep. 2015;11 (4):2592-2600. doi:10.3892/mmr.2014.3051

34. Munoz-Schuffenegger P, Ng S, Dawson LA. Radiation-induced liver toxicity. Semin Radiat Oncol. 2017;27(4):350-357. doi:10.1016/j. semradonc.2017.04.002

35. Imaeda M, Ishikawa H, Yoshida $Y$, et al. Long-term pathological and immunohistochemical features in the liver after intraoperative whole-liver irradiation in rats. $J$ Radiat Res. 2014;55(4):665-673. doi:10.1093/jrr/rru005
36. Zhao JD, Jiang GL, Hu WG, Xu ZY, Wang CF. Hepatocyte regeneration after partial liver irradiation in rats. Exp Toxicol Pathol. 2009;61 (5):511-518. doi:10.1016/j.etp.2009.02.114

\section{Publish your work in this journal}

OncoTargets and Therapy is an international, peer-reviewed, open access journal focusing on the pathological basis of all cancers, potential targets for therapy and treatment protocols employed to improve the management of cancer patients. The journal also focuses on the impact of management programs and new therapeutic

Submit your manuscript here: https://www.dovepress.com/oncotargets-and-therapy-journal agents and protocols on patient perspectives such as quality of life, adherence and satisfaction. The manuscript management system is completely online and includes a very quick and fair peer-review system, which is all easy to use. Visit http://www.dovepress.com/ testimonials.php to read real quotes from published authors. 